\title{
A note of thanks
}

\section{Albert Hofman ${ }^{1}$}

Published online: 30 November 2017

(C) Springer Science+Business Media B.V., part of Springer Nature 2017

The editor-in-chief, editors, members of the editorial board and publisher of the European Journal of Epidemiology wish to express their sincere gratitude to the referees of the articles considered for the 2017 volume of the Journal.

Hieab Adams

Anders Ahlbom

Olof Akre

Alvaro Alonso

Annick Alperovitch

Ubai Alsharif

André Amaral

Najaf Amin

Marzieh Araghi

Oscar Aramburu Bodas

Alberto Ascherio

Bjørn Åsvold

Henri-Jean Aubin

Dagfinn Aune

Cristina Baena

Fabian Bamberg

Christina Bamia

Rui Baptista

Olga Basso

Ettore Beghi

Gundula Behrens

Ulla Beijer

Jane Bell

Amy Bender

Vassiliki Benetou

Valerie Beral

Anita Berglund

Alain G. Bertoni

Annelies Bildt

Marta Blangiardo

Stefania Boccia

Paolo Boffetta

Carolina Bonilla

$\triangle$ Albert Hofman

ahofman@hsph.harvard.edu

1 Boston, MA, USA
Sephanie Bonn

Charles Boucher

Kim Braun

Ralph Brinks

Zoe Broere-Brown

Guy Brusselle

Tim Byers

Cristina Canova

Marion Carayol

Giriraj Chandak

Ching-Yu Cheng

Jan-Willem W. Coebergh

Nancy Cook

Alessio Crippa

Darren Dahly

Jean Francois Dartigues

Sirwan Darweesh

George Davey Smith

Daniel Davis

Paulus de Jong

Bianca De Stavola

Abbas Dehghan

Olaf Dekkers

Jeroen Demmers

Ivonne Derks

Klodian Dhana

Ming Ding

Monica Dinu

John Donohue

Mary K. Downer

Kathleen Egan

Hanan El Marroun

Alexis Elbaz

Maria Elmberg

Jutta Engel

Janine Felix

Bart Ferket

W. Dana Flanders 
Renee Fortner

Paul Foster

Rosanne Freak-Poli

Fernando Frioes

Silvano Gallus

Xiaoyi Gao

A. Garcia Rasio

Akhgar Ghassabian

Mika Gissler

Jean Golding

Kirstin Greaves-Lord

Sander Greenland

Oskar Hagberg

Anna Hansell

Jan Heeringa

Michael Hengartner

Christian Herder

Michael Hoffmeister

Bernd Holleczek

Lifang $\mathrm{Hou}$

Markus Huebscher

Adam Hulman

David Hunter

Mohammad Ikram

David Jacobs

Pauline Jansen

Loes Jaspers

Nicholas Jewell

Susan S. Jick

Michel Joosten

Maryam Kavousi

Niels Keiding

Jessica Kiefte-de Jong

Leo Kinlen

Manolis Kogevinas

Sarah Kölling

Chantal Koolhaas

Bernd Kowall

Gerard Krause

Lewis Kuller

Elzbieta Kuzma

Ilias Kyriopoulos

Carlo La Vecchia

Arnaud Lafon

Susanna Larsson

Graham Law

Joseph H. Lee

Michael Leitzmann

Maria Leon

Emmanuel Lesaffre

Jun Z. Li

Erikka Loftfield

Giancarlo Logroscino
Koos Lubsen

Lise Lund Haheim

Miguel Angel Luque Fernandez

Anubha Mahajan

Orly Manor

Mohammad Ali Mansournia

Manuela Marron

Miguel Ángel Martínez

Angelique Mavrodaris

Matthias Möhner

Ute Mons

Aline Moraes

Taulant Muka

Donald Mutti

Jana Nano

Katharina Nimptsch

Mireia Obón-Santacana

Shuji Ogino

Tomoyuki Ohara

Jorn Olsen

Sjurdur Frodi Olsen

Philip Orfanos

Toshiaki Otsuka

Eleni Papadopoulou

Frederik Peters

Ruth Peters

Jessica Petrick

Andreas Pettersson

Marina Pinhero

Laura Pletsch Borba

Bruce M. Psaty

Chengxuan Qiu

Sabah Quraishi

Francesco Raso

Roland Rau

Olivia Remes

Kjetil Retterstøl

Edo Richard

Brent Richards

Lorenzo Richiardi

Jolien Rijlaarsdam

Dimitris Rizopoulos

Meucci Rodrigo

Jeanine Roeters van Lennep

Lyda Rojas Sanchez

Frits Rosendaal

Kenneth Rothman

Oscar Rueda

Heba Sabbagh

Marc Saez

Jonathan M. Samet

Hanen Samouda

Susana Santos 
Sarah Schalekamp-Timmermans

Tania Schink

Carsten Schmidt

Josje Schoufour

Miranda Schram

Joachim Schuz

Eric Sijbrands

Cheikh Sokhna

Henrik Sørensen

Manon Spaander

Andreas Stang

Eric Steegers

Ewout Steyerberg

Bjørn Strand

Sonja Swanson

Moyses Szklo

Dirk Taeger

Masataka Taguri

Roy Thurik

Lau Thygesen

Hervé Tissot-Dupont

Eleonora Tobaldini

Britton Trabert

Jessica Tyrrell

Linda Valeri
Ed F. van Beeck

Nick van Es

Pim van Gool

Astrid van Hylckama Vlieg

Frank van Rooij

Lenie van Rossem

Andre L. M. Verbeek

Marina Vercelli

Elize Verhoeff

Virginie Verhoeven

Alexander Viktorin

Nadia Vilahur

Ellis Voerman

Trudy Voortman

Molin Wang

Lan Wen

Walter Willett

Renate Winkels

Frank Wolters

Angela Wood

Fei Yang

Hiroshi Yokomichi

Hajo Zeeb

Qingying Zhang 